\title{
GT2020-14927
}

\section{SUPER AGGRESSIVE S-DUCTS FOR AIR BREATHING ROCKET ENGINES}

\author{
J.V. Taylor, F. Flanagan, A. Dunlop, S.D. Grimshaw, R.J. Miller \\ Whittle Laboratory, University of Cambridge, United Kingdom \\ Email: jvt24@cam.ac.uk
}

\begin{abstract}
Air breathing rocket engines require turbomachinery and ducting that is substantially lighter than that used in ground based or aerospace gas turbines. In order to reduce the weight of the axial compressor, the design of the inter-spool swan neck duct is targeted. In this paper a circumferential splitter blade is used to reduce loading and diffusion on the duct endwalls. The splitter and duct geometry are coupled and optimised together using $2 D$ CFD. A design is selected that is $30 \%$ shorter than ducts that are currently used in aerospace gas turbines and the $3 D$ flow features are investigated in further detail using an experimental rig and $3 D C F D$.

This paper shows that the "splittered" duct has 3 benefits over a conventional duct design: First, separation of the endwalls is prevented even at short duct lengths, this will reduce distortion into the downstream compressor. Second, losses generated by corner separations on structural struts can be reduced by $20 \%$, enabling short ducts to achieve high performance. Third, splittered ducts are shown to be twice as robust to uncertain inlet flow conditions as conventional ducts. This allows a designer to target high performance short designs with reduced risk.
\end{abstract}

\section{INTRODUCTION}

The Synergetic Air-Breathing Rocket Engine (SABRE) is being developed by Reaction Engines Limited (REL) for use with a single stage to orbit vehicle. In air-breathing mode high pressure air is fed to the SABRE rocket combustion chamber via an intake, air pre-cooler and turbo-compressor. At speeds in excess of Mach 5, the intake is closed and the engine switches to operation as a conventional high-performance rocket which can accelerate the vehicle to orbital speed [1] [2].

In a space application, minimising engine mass is essential; for example, an engine mass saving of $1 \mathrm{~kg}$ reduces fuel consumption by $10 \mathrm{~kg}$ for a system with a propellant mass fraction of 0.9 . The ducting used to package the turbomachinery inside SABRE is critical in determining the overall length and therefore weight of the engine. In this work, the S-duct between the intermediate and high pressure compressors is studied. This duct is necessary to change the radius

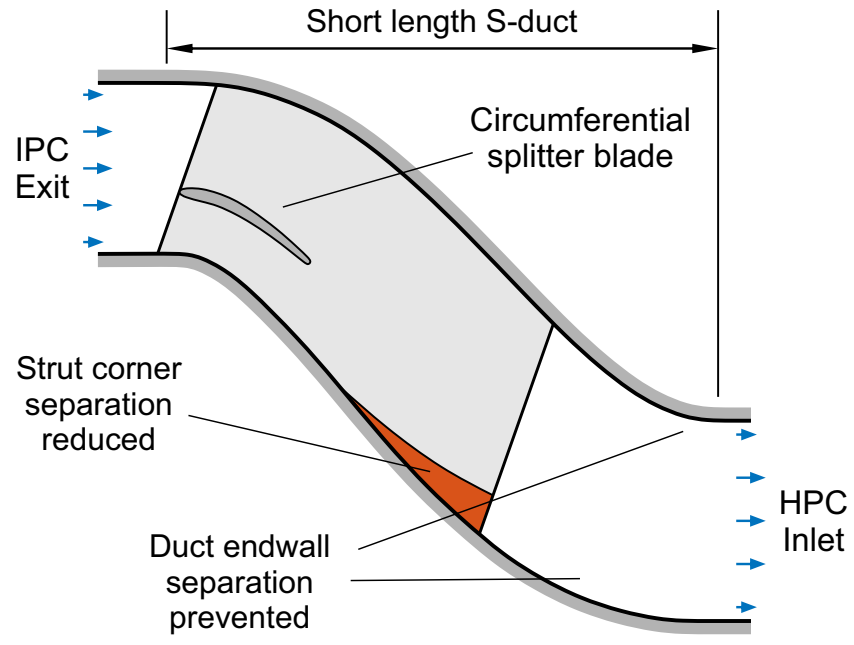

FIGURE 1: Using a splitter blade to prevent strut and endwall separation in short S-duct designs

of the flow between compressors and reducing its length reduces the weight to the shaft and casings.

The aim of this paper is to minimise S-duct length through the addition of a circumferential blade or "splitter". The meridional view of the duct and splitter blade is shown in Figure 1. In this design the splitter blade helps turn the flow through the radius change, it reduces the loading and the diffusion that is carried by the duct endwalls themselves. This prevents separation that can occur on the hub surface on the first bend and the casing surface on the second bend. The splitter can also be used to reduce the size of the corner separations that occur on struts that are structural or necessary to pass services through the gas path. This is achieved by reducing both the radial pressure gradient imposed on the strut boundary layer and the diffusion carried by the hub. Splitter blades are used successfully in ducting to package combustion chambers or radial turbomachines, however they have not been used in a published inter-spool S-duct before. Not only does this paper show that they will probably be necessary in a space application, but also that they could be useful in an aerospace gas turbine. 


\section{LITERATURE REVIEW}

S-duct curvature creates pressure gradients normal to the streamlines and these lead to adverse pressure gradients on the hub side of the first bend and casing side of the second bend. The level of curvature, and hence strength of adverse pressure gradients, is characterised by $\Delta R / L$. Increasing this parameter produces shorter and lighter S-ducts which are said to have increased "loading" and be more "aggressive" due to the stronger pressure gradients produced. Streamwise pressure gradients can also occur due to changes in area through the S-duct and this effect is represented by the ratio of exit to inlet area, $A_{\text {out }} / A_{\text {in }}$. Typically, however, area change is small and in most published studies the inlet-to-exit area ratio is equal to 1, e.g. [3], [4], [5].

S-duct designs must avoid endwall separations because they cause an unacceptable increase in loss while also reducing the performance of downstream compressor stages. Early S-duct studies (e.g. [6], [7]) describe cases with low loading where $\Delta R / L$ is less than 0.3 and there are no separations. Reducing S-duct length (or increasing the change in radius) increases the end wall adverse pressure gradients and a limit of $\Delta R / L$ is reached where the flow on either the hub or casing separates. Ortiz Dueñas et al. [3] studied this effect. A datum S-duct with no-struts, designed to have loading representative of a modern aerospace gas turbine $(\Delta R / L=0.5$, $A_{\text {out }} / A_{\text {in }}=1$ ), was tested experimentally and found to have stagnation pressure loss coefficient, $Y_{p}=0.041 . \Delta R / L$ was then increased to 0.67 by reducing the duct length while also re-optimising the turning angle at the geometric centre of the duct; this increased $Y_{p}$ to 0.052 . Further increasing $\Delta R / L$ to 0.78 (also with re-optimisation) is shown to cause endwall separation and an increase in loss to $Y_{p}=0.128$.

To reduce overall length Walker et al. [5] describe how upstream compressor outlet guide vanes (OGVs) can be moved into the first bend of an S-duct with no struts. New OGVs were designed with tangential lean and axial sweep to match the static pressure field of the S-duct. The new, shortened design is shown to have minimal impact on the upstream rotor row or the flow at exit of the S-duct. However, the use of OGV lean to change the radial static pressure gradient and modify the end wall loading is not demonstrated. In another paper Walker et al. [8] further modify their design by adding a bleed slot to the S-duct hub. This design with integrated OGV, 5\% bleed from the hub and no strut, has $\Delta R / L=0.59$.

Large, non-lifting, structural struts which pass services to and from the hub often intersect the S-shaped duct. These are characterised by thickness-to-chord ratio, $t / c$, which is typically between 0.2 and 0.3 for modern aero-engines [4]. Flow past the strut is accelerated and then decelerated due to its thickness. At the hub, where the S-duct curvature already causes an adverse pressure gradient, the additional diffusion caused by the rear section of the strut can cause a corner separation to form.

The behaviour of S-ducts with struts has been studied by a number of researchers. Bailey et al. [7] show that the strut in their experiment had a significant effect on the duct's static pressure field. However, with $\Delta R / L=0.26$ and $t / c=0.12$, the endwall and strut loading was low and no separations occurred. Naylor et al. [4] investigated a highly loaded S-duct and strut with $\Delta R / L=0.67$ and $t / c=0.27$. Experimental measurements show that a large corner separation was formed between the strut and hub and the measured loss coefficient was $Y_{p}=0.090$. The same S-duct geometry without a strut was previously tested by Ortiz Dueñas and has $Y_{p}=0.052$. Inlet conditions can

\begin{tabular}{lc}
\hline$\Delta R / L$ & 0.66 \\
$R_{\text {in }, \text { hub }} / R_{\text {in }, \text { cas }}$ & 0.88 \\
$A_{\text {out }} / A_{\text {in }}$ & 1.00 \\
$A_{\text {out }} / A_{\text {min }}$ & 1.16 \\
Number of struts & 12 \\
Strut $t / c$ & $37 \%$ \\
Splitter $c / s$ & $87 \%$
\end{tabular}

TABLE 1: Design parameters of optimised splittered duct

also effect S-duct and strut performance. Sonoda et al. [9] show that duct loss increases by $33 \%$ as the inlet boundary layer thickness is increased from 5\% of span to 30\%. Bailey et al. [7] and Karakasis et al. [10] show that including an upstream compressor stage increases duct loss; this is principally due to the presence of OGV wakes.

Naylor et al. [4] applied non-axisymmetric endwall profiling, local to the strut, to remove the strut-hub corner separation. The endwall geometry was produced by numerical optimisation and the approach works by "area ruling" the passage close to the strut in order to reduce the local diffusion. The new design was tested experimentally and shown to completely remove the corner separation. A similar approach is adopted by Stürzebecher et al. [11] where a computational optimisation combines non-axisymmetric endwall profiling, OGVs integrated into the duct and a turning strut which builds on the work of Walker et al. [12] and Wallin et al. [13]. The resulting design is $19 \%$ shorter than an already aggressive baseline with no predicted increase in loss.

In this paper we introduce a new way to design aggressive S-ducts which are free of separations. A circumferential splitter blade is used to reduce the adverse pressure gradients on the highly loaded duct endwalls as shown in Figure 1. This approach is similar to that used by Clark et al. [14] where splitter vanes are used for secondary flow control in low aspect ratio turbine vanes. Clark demonstrates that it is necessary to optimise the turbine blade and splitter geometry together as they are strongly coupled.

\section{APPROACH}

Our approach breaks down into three parts. First, the design space of the "splittered" S-duct geometry is explored and 2D computational fluid dynamics (CFD) is used to find optimal designs for different duct lengths. Second, the three-dimensional flow mechanisms in an aggressive, optimised and splittered S-duct are investigated using an experimental rig and 3D CFD; the parameters of this design are given in Table 1. Third, 3D CFD is used to quantify the robustness of splittered duct designs to uncertain inlet flow conditions.

\section{Numerical Methods}

Computations are performed using TURBOSTREAM, a Reynoldsaveraged Navier-Stokes solver that runs multi-block, structured grids on GPUs (graphical processing units). The Spalart-Allmaras (SA) turbulence model is used with wall functions [15] and all cases are run to a steady state. TURBOSTREAM is described in more detail and validated in [16]. 


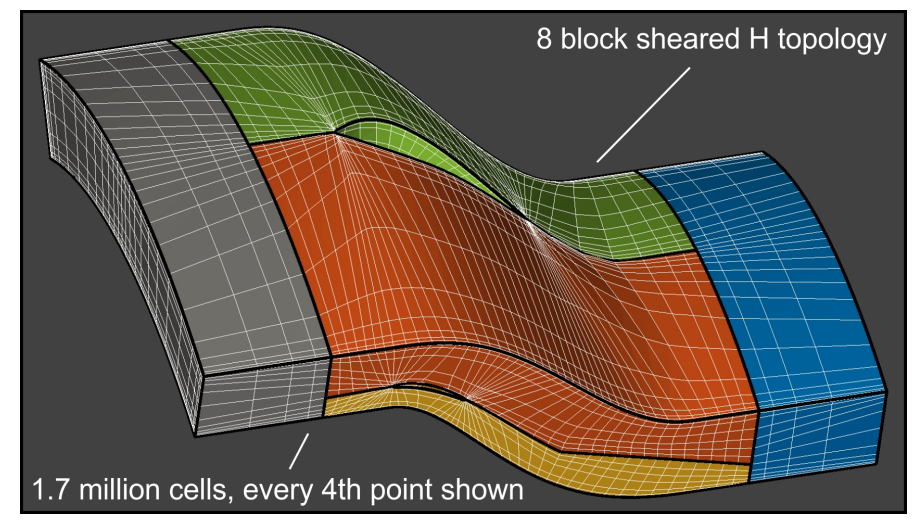

FIGURE 2: Configuration of the 3D duct and strut mesh

In the study of the splittered S-duct design space 2D axisymmetric CFD simulations are used to model the flow. A smoothed, sheared-H topology is used around the splitter blade with four blocks forming the inlet, the outlet, the block between splitter and casing and the block between splitter and hub. This meshing scheme was fully automated to account for changes in geometry during the optimisation procedure. As ducts are modified the number and distribution of nodes is automatically updated to ensure $y^{+} \approx 30$ is maintained on all surfaces. A typical number of cells in these 2D cases is 20,000 .

3D CFD simulations are performed to investigate the flow mechanisms in the experimentally tested splittered S-duct with strut. For this geometry the grid is modified from the 2D case to include four additional blocks which form the mesh around the strut. As with the splitter blades a smoothed sheared-H topology is used around the strut, the resulting mesh is shown in Figure 2. In this case 1,700,000 cells are used to fully resolve the real experimental geometry.

\section{Experimental Methods}

A modular experimental rig was designed to allow different endwall, strut and splitter configurations to be rapidly tested. The parts are 3D printed using FDM (fused deposition modelling) and the modular design provides easy access when surface flow visualisation photographs are required.

A $45^{\circ}$ sector was selected in order to maximise the Reynolds number of the tests given a limited air supply. The Reynolds number achieved is 140,000 based on the span of the duct inlet and 340,000 based on strut chord, the inlet Mach number is 0.19. A pull down configuration is used so that the inlet flow can be controlled into the sector and unwanted distortions avoided.

Boundary layer trips are used on the inlet hub and casing surfaces to trip the boundary layers to become fully turbulent and to accelerate their growth to $15 \%$ of the span. This target inlet profile was taken from the exit of a multi-stage calculation of an IPC. The exact CFD boundary condition was derived from measurements at inlet to the rig. The effect of different inlet flow profiles is investigated using CFD in the final section on uncertainty and robustness. No trips are applied to the sidewalls of the sector so that thin boundary layers are maintained on these surfaces. A turbulence grid is placed upstream of the duct to provide freestream turbulence levels of $2 \%$.

A miniature five-hole probe with head diameter $1 \mathrm{~mm}(2.6 \%$ span) [17] is used to perform area traverses at inlet and exit of the $\mathrm{S}$-duct. The probe is sealed in a rotating section of casing so that the

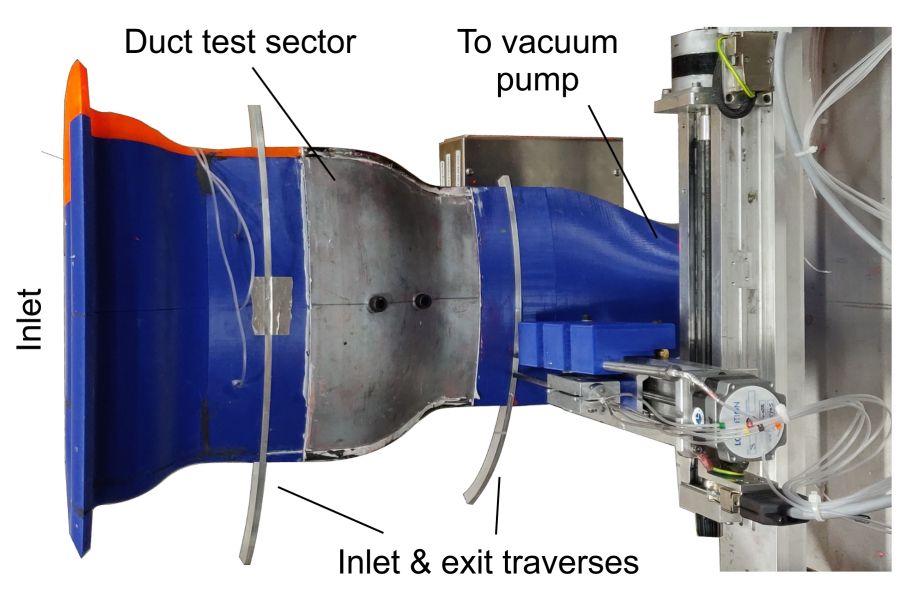

FIGURE 3: Configuration of the duct sector test rig

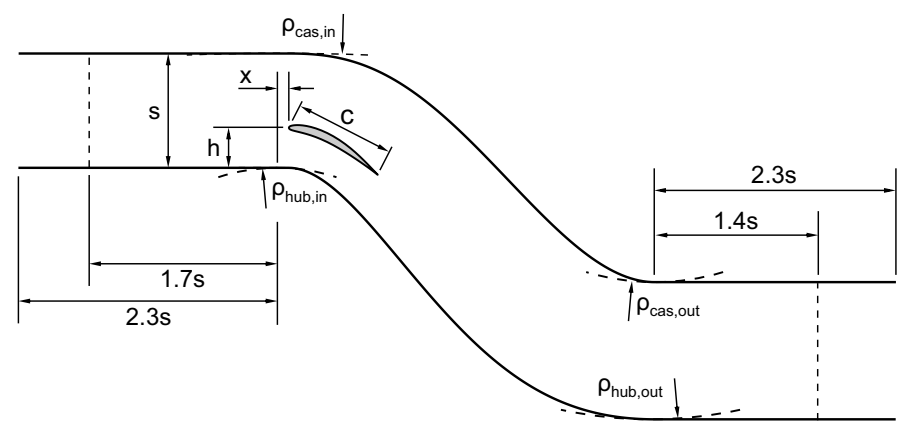

FIGURE 4: Parameterisation of the splittered duct and schematic of CFD domain

entire sector can be measured. A photograph of the rig and traverse setup is shown in Figure 3. In order to confirm the periodicity of the experiment the flow uniformity is tested at the duct exit with no splitter or strut installed. A five-hole probe traverse is performed over the $45^{\circ}$ sector and the flow is found to be axisymmetric about the central $30^{\circ}$ sector of the rig.

Area traverses at inlet and outlet are used to evaluate the stagnation pressure loss coefficient, Equation 1, for the duct designs tested in this paper. Pressures are mass averaged to determine 1D values and an identical method is used to calculate loss coefficients from the CFD results.

$$
Y_{p}=\frac{\overline{P_{o, \text { in }}}-P_{o, \text { out }}}{\overline{P_{o, \text { in }}}-\overline{P_{\text {in }}}}
$$

\section{SPLITTERED S-DUCT DESIGN SPACE}

In this section the coupled design of S-duct endwalls and splitter blade geometry is investigated. S-duct length is varied and for each value of $\Delta R / L$ the geometries of a conventional and splittered $S$-duct design are optimised. Performance is then compared between the two types of S-duct for a range of different length designs. At the end of this section an aggressive, splittered S-duct design is selected for validation in experiment and analysis with 3D CFD.

While exploring the splittered S-duct design space it helps to simplify the geometry from that of a real engine by removing the strut. The S-duct model is therefore axisymmetric and the computational 


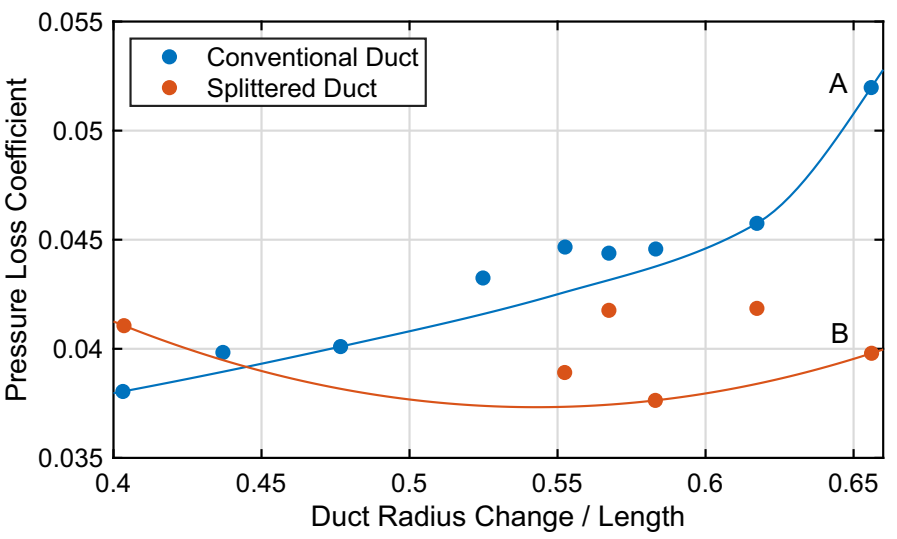

FIGURE 5: Computed loss coefficient of optimised conventional and splittered S-ducts at different lengths

power and time required to simulate the flow is significantly reduced. Using TURBOSTREAM, the geometry is solved in 2D cylindrical polar coordinates in 2 minutes on a single NVIDIA K20 GPU.

Figure 4 shows the domain of the S-duct. The inlet and exit ducts extend 2.3 spans upstream and downstream of the duct, at constant radius, so that the boundary conditions do not interfere with the solution of the flow in the curved part of the duct. Sampling planes for calculating loss are positioned 1.7 spans upstream of the first bend and 1.4 spans downstream of the second bend.

The TABU search method [18] is used to optimise conventional and splittered S-duct geometries over a range of lengths. The objective is to minimise the loss coefficient evaluated between inlet and exit sampling planes. For a given $\Delta R / L$, the conventional S-duct geometry is parameterised using four variables: the radii of curvature at inlet and exit to the duct on both hub and casing, $\rho_{\text {hub,in }}, \rho_{\text {cas }, \text { in }}$, $\rho_{\text {hub,out }}, \rho_{\text {cas,out }}$, as shown in Figure 4 . Between these points the endwall shapes are fixed using quintic polynomials. For the splittered S-duct, Figure 4 shows that three additional parameters are required to describe the geometry: the spanwise offset of the splitter leading edge, $h$, and the axial offset, $x$, fix the splitter position and the chord of the splitter, $c$, sets its size. The inlet and exit angles of the splitter blade are determined from the weighted average of the hub and casing lines at the axial location of the splitter leading and trailing edges. The turning and thickness distributions of the splitter are kept constant and are similar to those of a controlled diffusion blade used in a modern axial compressor.

The set of duct and splitter design parameters is small enough to allow the optimisation algorithm to move quickly around the design space while also remaining flexible enough to represent a wide range of S-duct and splitter shapes and combinations. TABU varies and tests each parameter independently so it is important that the parameterisation yields designs which are "sensible". It was found that controlling the S-duct endwalls with radii of curvature is more robust than other options, for example using coordinates of control points on splines.

Duct loading, $\Delta R / L$, is varied between 0.40 and 0.66 (n.b. modern aero engines currently operate around 0.5 [3]). Figure 5 shows the lowest stagnation pressure loss coefficient for the optimised conventional and splittered S-ducts at each length. The results are not smooth because the optimiser can get trapped in local minima and the markers therefore represent an upper bound on loss coefficient of the true optimal geometry. Polynomial curves were fitted through these

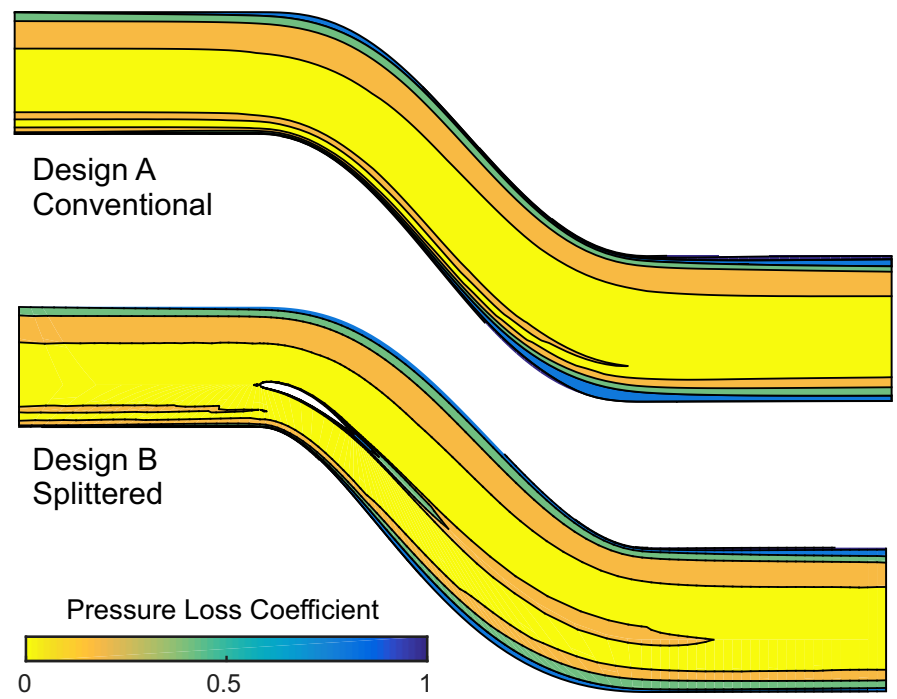

FIGURE 6: Computed loss coefficient of shortest designs for conventional duct (top) and splittered duct (bottom)

bounding points and have been added to the figure to illustrate what the relationship between fully optimised loss and length may look like.

Figure 5 shows that the loss coefficient for optimised conventional S-ducts increases with length and there is a steep increase for $\Delta R / L>0.62$; a similar result is reported in [4] for unstrutted, unsplittered ducts. For the splittered S-ducts, the loss coefficient varies by less than 0.002 over the entire range of $\Delta R / L$ and a possible minimum is indicated at $\Delta R / L=0.54$. This means that for long, conservative duct designs $(\Delta R / L<0.4)$, where endwall boundary layers remain attached, splitters offer no benefit; they only cause increased loss. However, for more aggressive S-ducts $(\Delta R / L>0.5)$, splittered designs outperform conventional geometries and at $\Delta R / L=0.66$ the splittered design has $23 \%$ lower loss than the conventional design.

The geometries and flow fields of the shortest optimised S-duct designs are shown in Figure 6. Design A is a conventional design and $\mathrm{B}$ includes a circumferential splitter blade, both have a $\Delta R / L=0.66$. In Design $B$ the optimiser has driven the splitter to the front of the duct, close to the hub. The splittered design also has tighter radii of curvature at the hub side on the first bend and at the casing side on the second bend. The contours in Figure 6 show that the splitter wake generates an extra loss source. However, Design B also has thinner hub and casing boundary layers than the conventional design and overall the loss coefficient for the splittered design is lower.

The endwall static pressure distributions for the conventional and splittered designs are shown in Figure 7 and can be used to explain why the optimisation process has produced Design B. The splitter blade offloads the hub on the first bend and even with a tighter radius of curvature the splitter acts to reduce the overspeed and resulting diffusion when compared to the conventional design. This allows the duct in Design B to open out to a larger area between the bends, as seen in Figure 6. The larger area has two benefits: First, the static pressure is increased and the velocity is reduced. This decreases the attached loss in the endwall boundary layers because loss is proportional to the flow velocity cubed [19]. Second, by contracting the area at the second bend, the diffusion on the casing side is reduced, preventing separation and reducing loss.

For all the different length splittered S-ducts shown in Figure 5, 


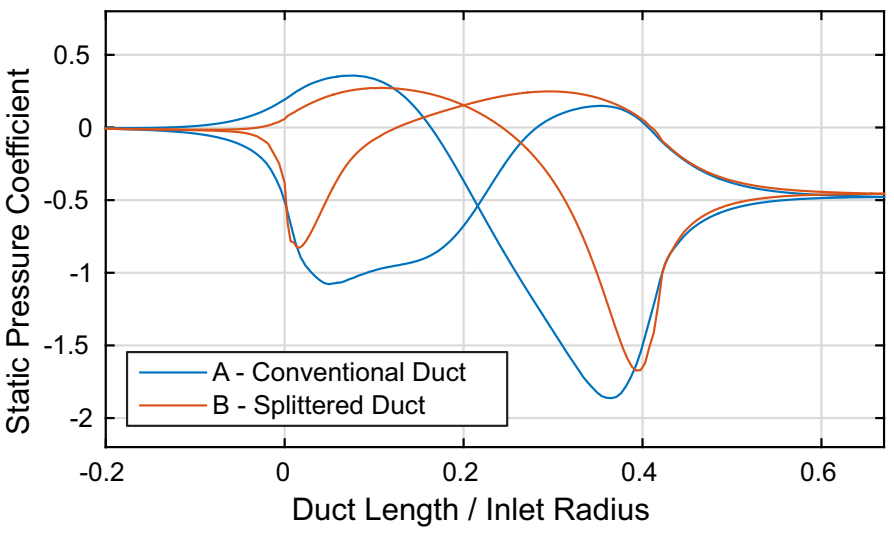

FIGURE 7: Computed static pressure coefficient on duct endwalls

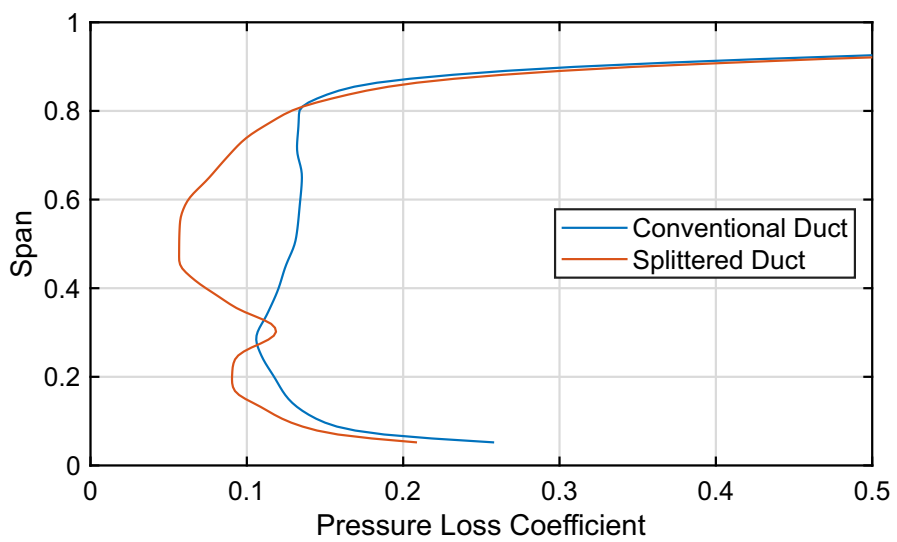

FIGURE 8: Experimental pressure loss coefficient profiles mass-averaged from area traverses at the duct exit

the optimisation process drives the splitter blade towards the front of the duct in order to offload the hub. This happens even when the optimiser is initialised with the splitter positioned at the back of the duct. The benefits of a splitter at the front of the S-duct are threefold: it prevents separation at the hub on the first bend by reducing diffusion, it reduces attached loss in the central region of the duct by lowering the velocity and it prevents separation at the casing on the second bend by contracting the duct area and re-accelerating the flow. Positioning the splitter blade at the back of the S-duct is not as effective since it can only reduce loss by unloading and preventing separation at the casing on the second bend.

For an air breathing rocket engine the most important factor to consider is its weight and this encourages the designer to use short, aggressive S-ducts. This section has shown that for short S-ducts an optimised, splittered design has $23 \%$ lower loss than an optimised conventional geometry. The shortest design investigated, Design B, is now taken forward for experimental testing and integration with a strut.

\section{D FLOW MECHANISMS}

This section describes the behaviour of splittered S-ducts which include structural struts, representative of a realistic engine geometry. The 3D flow mechanisms are studied and the performance of the splittered geometry is compared with a conventional design.

Figure 8 shows measured spanwise distributions of loss coefficient for the conventional and splittered designs at exit of the

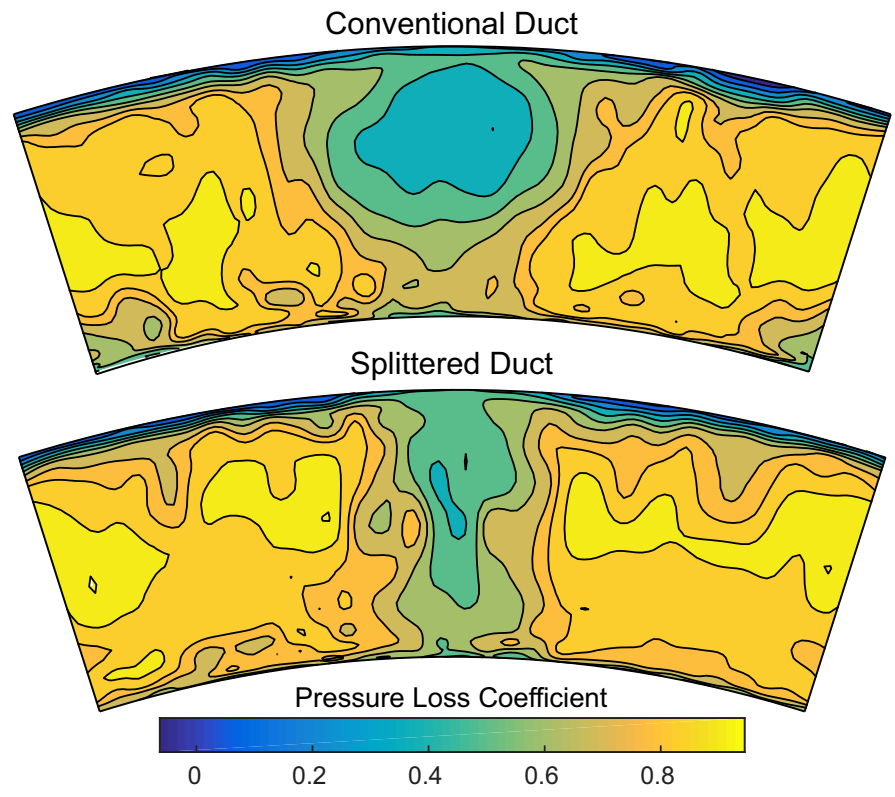

FIGURE 9: Experimental traverses at duct exit showing pressure loss coefficient of conventional duct (top) and splittered duct (bottom)

duct. These results are calculated from a pitch-wise mass average of the flow measured with five-hole probe area traverses, these are shown in Figure 9. The spanwise profiles in Figure 8 show that loss in the conventional design is greater than the splittered design close to the hub and over the outer $60 \%$ of span. The splitter wake persists to the exit traverse plane and is seen to cause an increase in loss at $30 \%$ span. The overall mass-averaged loss coefficient for the conventional design is 0.15 , while the splittered duct has a loss coefficient of 0.12 . These values are high compared to reported values in the literature [4] for three reasons: first, the S-duct is aggressive with $\Delta R / L=0.66$, second, due to the short duct the strut is thicker than usual with $t / c=37 \%$ and third, the periodic sector tested is $30^{\circ}$ so the number of struts per annulus is twelve.

Figure 9 shows that the strut causes most of the extra loss in the conventional design. The splitter blade reduces this loss in the region from 35 to $80 \%$ span downstream of the strut. The conventional design also has increased loss in two further places. Firstly Figure 9 shows the casing endwall boundary layers are thicker, this result also agrees with the 2D CFD in Figure 6. Secondly, there is an increase in loss at the hub midway between the struts in the conventional duct case. The only penalty in the splittered duct case is that it slightly increases the loss at $30 \%$ span across the whole pitch.

Surface flow visualisation was performed to investigate the origin of the loss sources in both conventional and splittered designs. Photographs of the oil streaks are shown in Figure 10. Both designs have corner separations between the strut and hub endwall surface, these are marked as A \& D in the photographs. For the conventional duct the corner separation is larger in terms of its spanwise penetration ( $\sim 60 \%$ span) and the chordwise position of its root $(\sim 20 \%$ strut chord). The annotation B shows a separation on the hub endwall surface in the conventional design. This does not extend across the entire pitch to the strut due to the blockage from both the strut and downstream corner separation. This blockage causes local acceleration of the flow, preventing separation in the vicinity of the strut itself. No hub endwall separation is present in the splittered duct design as 

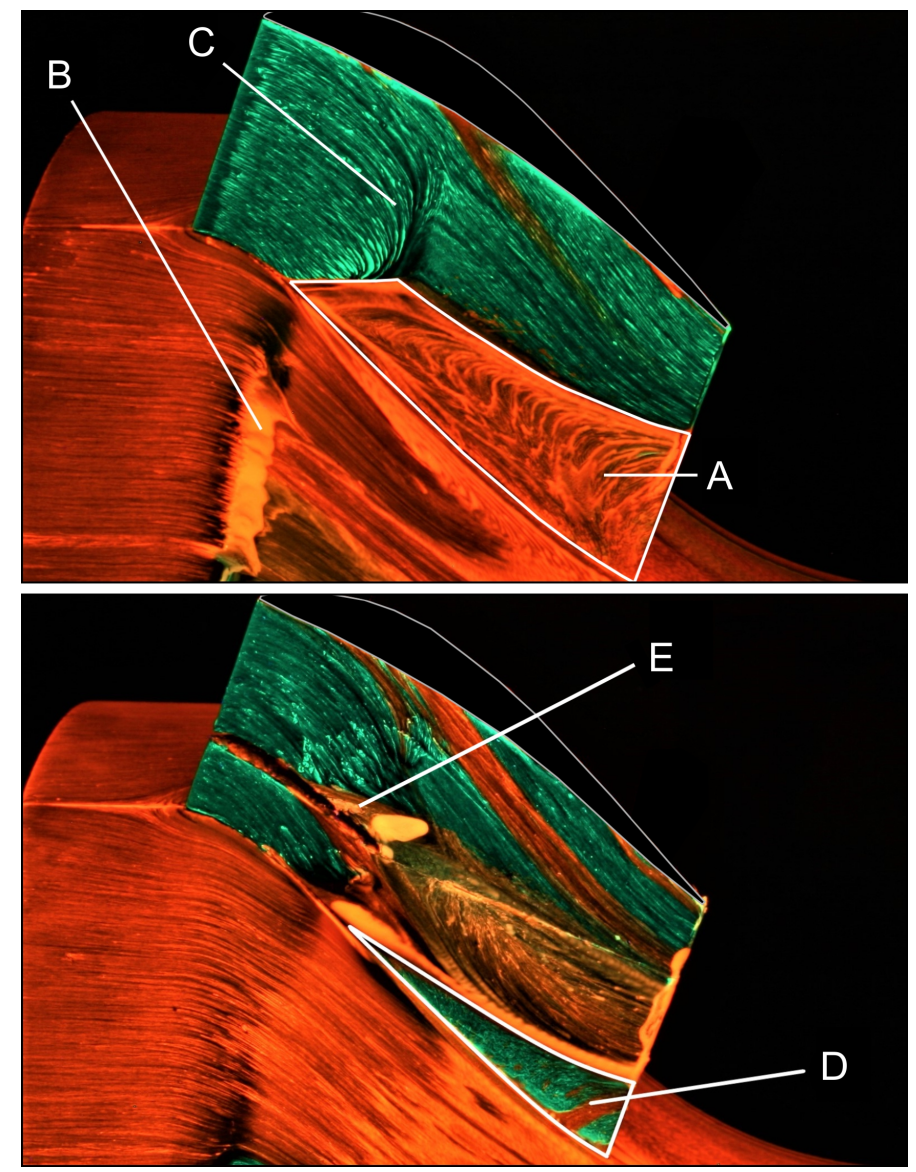

FIGURE 10: Experimental surface flow visualisation of strut and hub endwall of conventional duct (top) and splittered duct (bottom). The splitter blade is removed in the second photo.

\begin{tabular}{lcc} 
& Conventional Duct & Splittered Duct \\
\hline Experiment & 0.15 & 0.12 \\
3D CFD & 0.142 & 0.129
\end{tabular}

TABLE 2: Predicted and measured loss coefficients of duct designs

the splitter blade unloads the hub in the first bend. It is likely that B is the source of extra loss seen mid-pitch at the hub of the conventional duct in Figure 9 and in the mass averaged loss plotted in Figure 8.

E shows an additional corner separation where the suction surface of the splitter blade meets the strut. While this is an extra loss source not present in the conventional design it is a small feature and is outweighed by the benefits of reducing the large corner separation A. The effect of the corner separation at $\mathrm{E}$ is difficult to discern in Figure 9 as it is mixed in with the loss caused by the rest of the corner separation $\mathrm{D}$ and the strut wake. In the conventional duct design a separation line and reattachment can be seen at $\mathrm{C}$, this is caused by transition of the strut boundary layer aft of its maximum thickness location.

To further investigate the development of loss through the S-ducts, the conventional and splittered designs are simulated using 3D CFD. The predicted loss coefficients for CFD and experiments are compared in Table 2. The CFD predicts the splittered design to have $9.2 \%$ lower loss than the conventional design. This is less than the $20 \%$ benefit measured in the experiment primarily due to

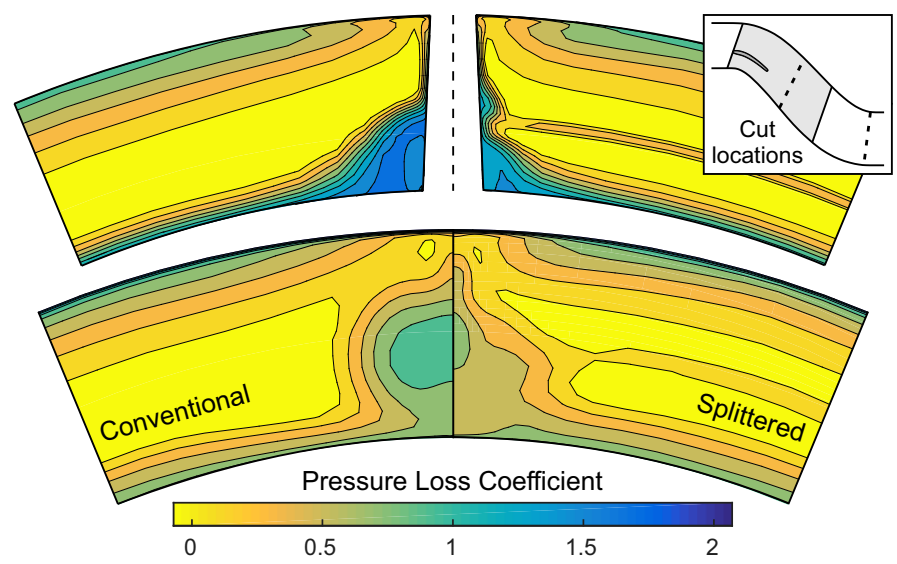

FIGURE 11: Computed loss coefficient at two locations mid duct for conventional ducts (left) and splittered ducts (right)

errors in the prediction of the size of the corner separation. However, it is instructive to use the CFD to investigate the origin of the flow structures and loss generating mechanisms.

Cuts are taken through the CFD solutions and loss coefficient is plotted in Figure 11. The cuts are taken at $60 \%$ strut chord and at the second bend of the duct as shown in the figure inset. In the conventional duct, at the first cut plane, the corner separation extends across $60 \%$ of span and a similar distance out into the passage. As this low stagnation pressure flow enters the second bend it is overturned by the spanwise pressure gradient and the loss core is pushed up the span away from the hub, as can be seen in the second CFD cut and the duct exit traverse in Figure 9.

In the splittered S-duct design the corner separation between strut and hub wall is reduced to around 30\% span. Another small corner separation, between strut and splitter blade is present at around $50 \%$ span. For this case the stagnation pressure deficit is not as great as in the conventional design. Therefore, the overturning caused by the spanwise pressure gradient, is reduced and the high loss region remains closer to the hub endwall downstream of the strut.

The experimental results and CFD both show that the flow through a splittered S-duct is extremely three-dimensional. Corner separations exist on multiple surfaces and strong pressure gradients, caused by turning of the duct endwalls, generate strong secondary flow. In this environment it is difficult to imagine an axisymmetric splitter being the optimal geometry in much the same way that axisymmetric endwalls were shown not to be optimal by Naylor et al. [4]. To investigate this idea a further experiment was performed with cropped splitter blades as shown in the inset in Figure 12. The aim of these tests is to study whether it is possible to retain the benefit of reducing the strut corner separation while avoiding the parasitic loss of splitter wakes away from the strut.

Splitter aspect ratio is reduced from a value of 7.0, which represents a complete uninterrupted splitter ring, down to 1.5. Area traverses were performed for each geometry and the mass-averaged overall loss coefficient is calculated and plotted in Figure 12. A reduction of splitter aspect ratio to 5.3 reduces loss by $11 \%$. Beyond this, as the splitter blades are cropped further, the loss begins to increase. This is caused by the combination of increased hub loss due to endwall separation and a new loss mechanism, the tip flow caused by the cantilevered splitter blade. 


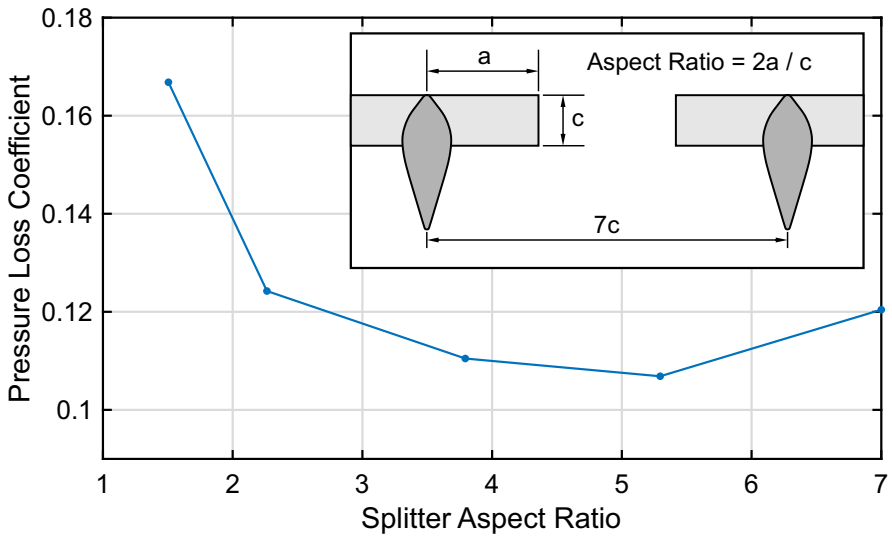

FIGURE 12: Experimentally determined pressure loss coefficient for cropped splitters of varying aspect ratio

While this approach has a benefit in terms of loss reduction, it is unlikely to be used in a real machine. A splitter of aspect ratio 5.3 is a relatively long blade to cantilever out from the strut and will likely encounter mechanical or vibration issues. However, these results do indicate that a non-axisymmetric splitter blade design could have significant benefit. One way to realise this in a practical application is to tailor the splitter blade chord length and turning across the pitch. This way the loss can be reduced midway between the struts where splitter loading can be safely decreased.

Circumferential splitter blades show great potential for use in aggressive S-ducts with structural struts. They have been shown to reduce the size of the hub side corner separation on the strut and completely prevent separation on the hub endwall. Combined, these effects result in $20 \%$ reduced loss. With non-axisymmetric tailoring of the splitter blade it is possible to reduce these losses further.

\section{UNCERTAINTY \& ROBUSTNESS}

S-duct design is often conservative because the inlet flow is of poor quality and has high uncertainty. Unlike compressor or turbine blades, which form a new boundary layer at each leading edge, an S-duct must turn flow that already has large, degraded endwall boundary layers caused by upstream turbomachinery. A large degree of uncertainty also exists around the size and condition of these boundary layers; a designer often has to rely on multi-stage CFD calculations of the upstream turbomachinery where cumulative errors are likely to build up from stage to stage. For an S-duct design to be successful it must maintain its performance even when operating with an inlet flow that is very different to the design intent. This section aims to quantify the robustness of conventional and splittered S-duct designs to large variations in inlet conditions.

The conventional and splittered duct designs, with structural struts, are run in 3D CFD with a range of different inlet flow conditions. 46 cases are run for each design with independently varied hub and casing endwall boundary layer size. The spanwise extent of the hub and casing stagnation pressure deficit, applied at inlet of the CFD domain, varies between $1.0 \%$ and $70 \%$. Examples of seven of these stagnation pressure profiles are shown in Figure 13. To compare results consistently, the hub and casing boundary layer displacement thickness is calculated at the inlet sampling plane for each case and is found to vary between $1.0 \%$ and $11 \%$ of span.

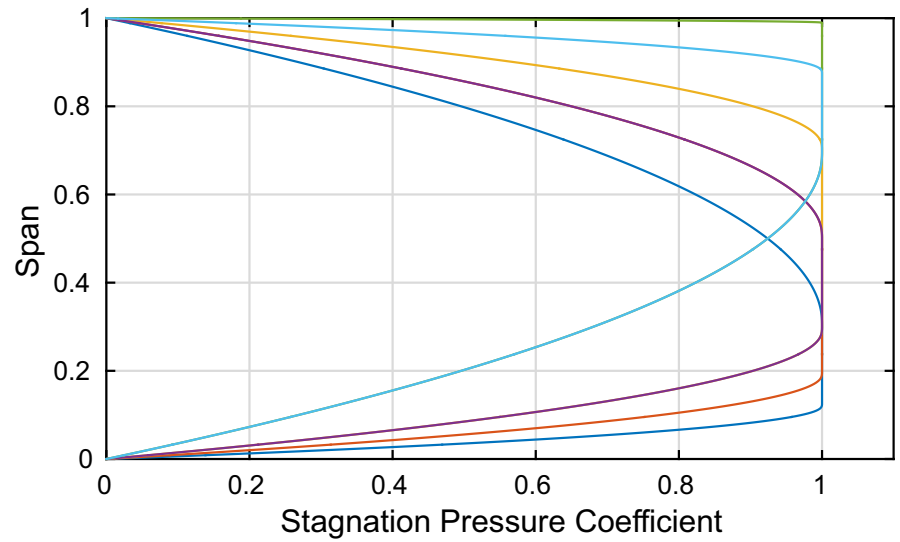

FIGURE 13: Seven examples of the stagnation pressure profiles applied at CFD domain inlet

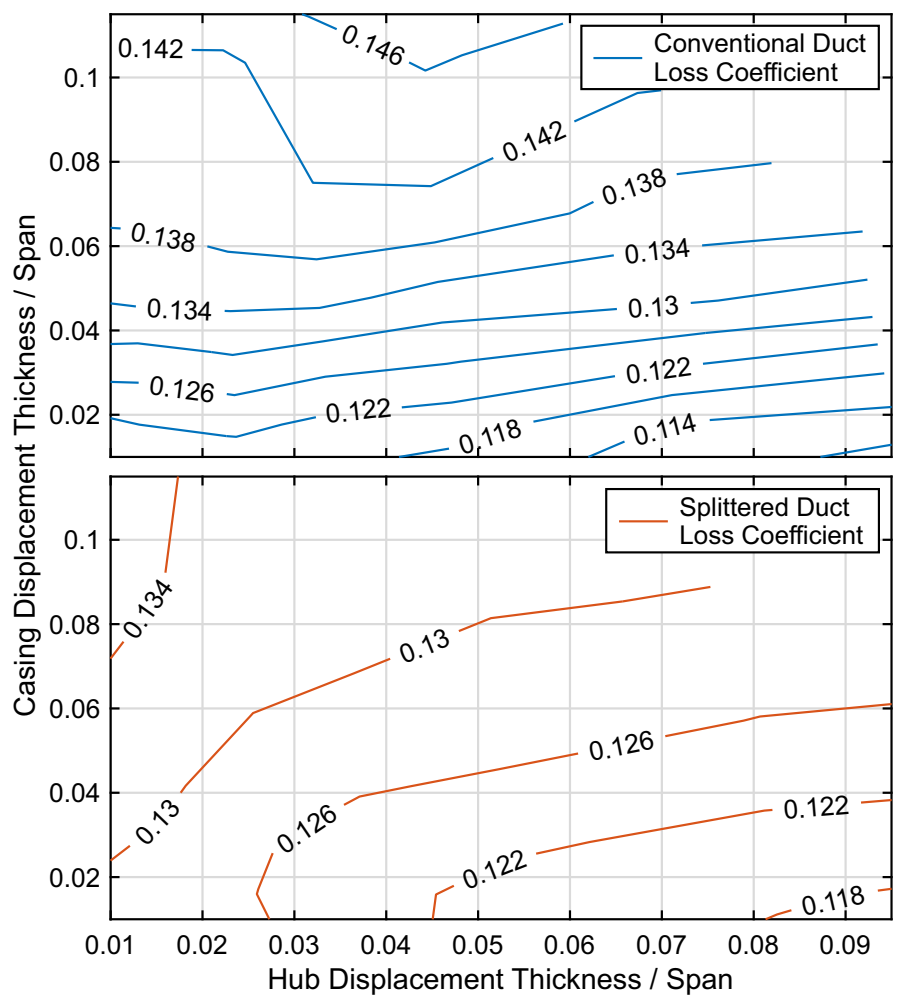

FIGURE 14: Computed pressure loss coefficient of conventional duct (top) and splittered duct (bottom) with varying hub and casing inlet endwall boundary layer thicknesses

The results of the robustness study are shown in Figure 14 where contours of loss coefficient are plotted for different inlet flow conditions with varying hub and casing displacement thickness. For the splittered duct design, the space investigated is covered by 5 contour levels, equivalent to a change in loss coefficient of 0.02 . In the case of the conventional duct design, 10 contour levels cover the space, a change in loss coefficient of 0.04. This shows that the sensitivity of the splittered duct design to inlet endwall boundary layer size is half that of the conventional design.

For a few inlet profiles, when the casing boundary layers are less than $3 \%$ of span, it can be seen that the conventional duct design outperforms the splittered design. In these cases the thin boundary 


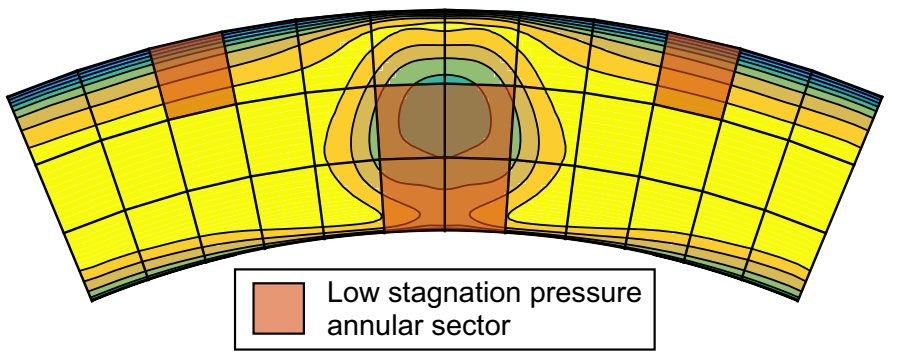

FIGURE 15: Calculation of distortion coefficient at duct exit

layer results in less diffusion on the casing endwall and it is possible to achieve good performance in a short duct without a splitter blade.

For the conventional design, Figure 14 shows that most of the contour lines are aligned with the $\mathrm{x}$-axis, meaning that loss coefficient is broadly independent of the size of the hub endwall boundary layer. This is because separations at the hub on the first bend are likely to reattach due to the strong favourable pressure gradient generated by the second bend. However, the casing boundary layer causes separation on the second bend that does not reattach and this results in extra loss. For the splittered design, contours of loss coefficient turn towards the vertical and spread out when the casing boundary layer displacement thickness is greater than $8 \%$ span. This shows that the tolerance of the S-duct to large casing boundary layers has been improved thanks to the "protective" mechanism of opening out the area in the central region of the duct and then contracting back down to accelerate the flow around the second bend.

Not only do unexpected separations in the duct cause extra loss, they also cause distortion and problems in the downstream turbomachinery. The 46 cases with different inlet profile sizes are analysed and the distortion coefficient $(D C)$ described in Equation 2 is calculated at duct exit. To evaluate this parameter, the flowfield at the exit sampling plane is subdivided into 3 spanwise and 12 pitchwise annular sectors, these are shown for an example case in Figure 15. In each sector the stagnation pressure is area averaged and the 6 lowest values are calculated. The mean of these 6 values then gives the value of $P_{o, \text { out,min }}$. This treatment is based upon the $D C 60$ parameter used in [20]. However, the new method is more suitable in this application as it takes account of both hub and casing endwall separations as well as growth of the corner separations on the strut itself.

$$
D C=\frac{\overline{P_{o, \text { out }}}-P_{o, \text { out }, \text { min }}}{\overline{P_{o, \text { in }}}-\overline{P_{\text {in }}}}
$$

Figure 16 shows contours of distortion coefficient calculated for varying inlet boundary layer thickness for both conventional and splittered duct designs. It shows that the splittered duct design has a lower distortion coefficient across the entire range of inlet conditions tested, with a mean value $15 \%$ lower than the conventional design. The distortion at exit of the splittered duct is also less sensitive, the space is covered by 7 contour levels, equivalent to a change in $D C$ of 0.12 . For the conventional duct the space is covered by 9 contour levels, a change in $D C$ of 0.16 .

Duct design in aero engines is conservative as separations due to uncertain inlet flow conditions can result in significant loss as well as problems for downstream turbomachinery; design in a hybrid engine for space access is expected to be just as challenging. A splittered S-duct is twice as robust to varying inlet conditions as a conventional

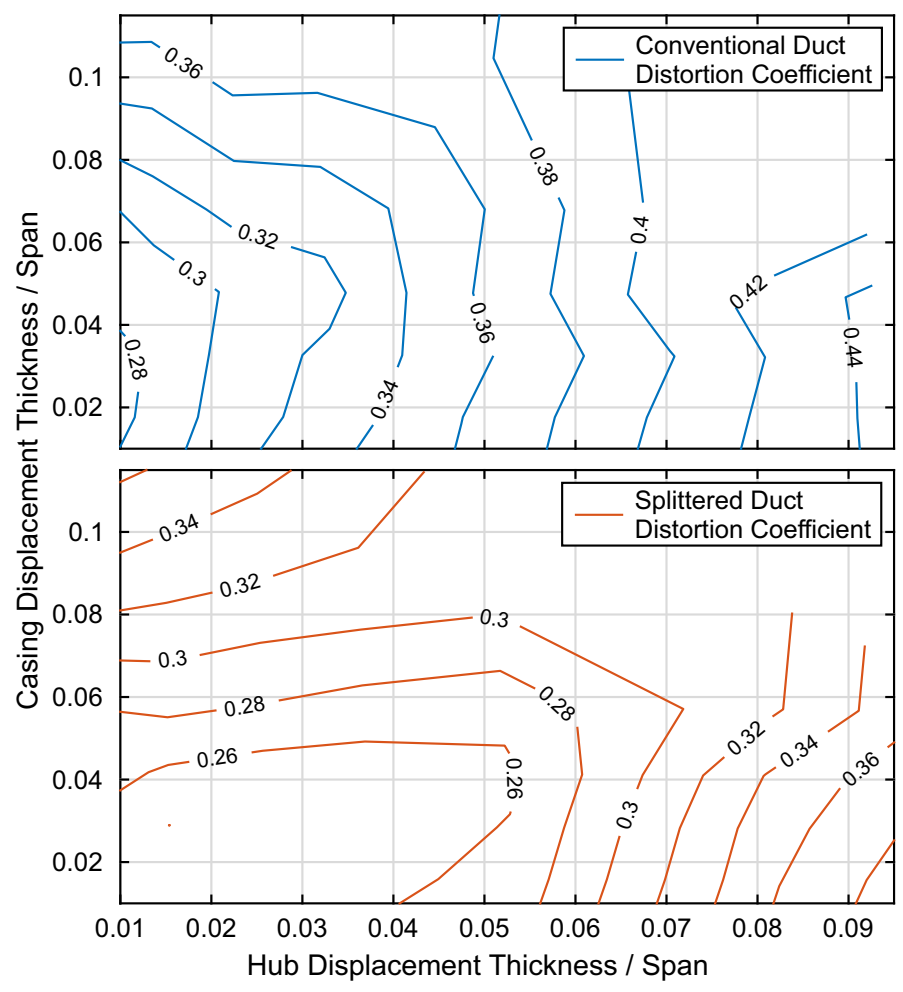

FIGURE 16: Computed distortion coefficient of conventional duct (top) and splittered duct (bottom) with varying hub and casing inlet endwall boundary layer thicknesses

design, this technology is a promising method by which a designer can reduce risk in their engine development programme.

\section{CONCLUSIONS}

In a hybrid air breathing rocket engine reduced weight is the most important objective. This means that technologies may be considered which have higher loss than those used in aero engine gas turbines if they can save significant engine weight. The weight saving technology investigated in this paper is the use of a circumferential splitter blade to enable shorter S-duct designs.

2D optimisation of the coupled S-duct endwall and splitter geometry shows that aggressive S-duct designs $(\Delta R / L>0.5)$ with splitter blades have lower loss than conventional designs. For $\Delta R / L=0.66$ an optimised splittered design has $23 \%$ lower loss than an optimised conventional geometry.

3D CFD and experiments show that in aggressive S-ducts with structural struts the flow is highly three-dimensional with corner separations on multiple surfaces and strong secondary flows. In this environment the splittered design outperforms a conventional duct and loss is reduced by $20 \%$ due mainly to the reduction in size of the strut-hub corner separation.

In theory, the goal of S-duct design is to reduce length, however, in practice designers are most concerned about risk. Duct lengths increase because attached flow has to be maintained even with uncertain duct inlet flow conditions. 3D CFD simulations show that splittered duct designs are twice as robust to uncertainty than conventional designs so should be the first technology a designer uses to reduce engine weight in a high performance space application. 


\section{NOMENCLATURE}

A Duct area

a Splitter tangential length

$c \quad$ Chord

$D C$ Distortion Coefficient

$h \quad$ Splitter spanwise offset

$L \quad$ Duct length

$P \quad$ Pressure

$s \quad$ Span

$t \quad$ Strut thickness

$x \quad$ Splitter streamwise offset

$Y_{p} \quad$ Pressure loss coefficient

$\Delta R \quad$ Midspan duct radius change

$\rho \quad$ Radius of curvature

\section{ACKNOWLEDGEMENTS}

The authors would like to thank Reaction Engines Ltd. for their contribution to this work and TURBOSTREAM for the use of their solver. They are also grateful for the comments and suggestions of colleagues at the Whittle Laboratory and Reaction Engines Ltd. Most notably Chris Clark, Osama Lotfi, Kathryn Evans and Marc Arstall.

\section{REFERENCES}

[1] Bond, A., and Varvill, R., 2018. Engine comprising a rocket combustion chamber and a heat exchanger, July 3 . US Patent $10,012,177$.

[2] Bond, A., 2018. Rotational machine, such as a turbomachine, and engines and flying machines incorporating same, Aug. 28. US Patent App. 10/060,356.

[3] Ortiz Duenas, C., Miller, R. J., Hodson, H. P., and Longley, J. P., 2007. "Effect of length on compressor inter-stage duct performance". Vol. GT2007-27752 Volume 6: Turbo Expo 2007, Parts A and B of Turbo Expo: Power for Land, Sea, and Air, pp. 319-329.

[4] Naylor, E. M., Dueñas, C. O., Miller, R. J., and Hodson, H. P., 2010. "Optimization of nonaxisymmetric endwalls in compressor s-shaped ducts". Journal of Turbomachinery, 132(1), p. 011011.

[5] Walker, A. D., Barker, A., Carrotte, J. F., Bolger, J., and Green, M., 2013. "Integrated outlet guide vane design for an aggressive s-shaped compressor transition duct". Journal of Turbomachinery, 135(1), p. 011035.

[6] Britchford, K., Manners, A., McGuirk, J., and Stevens, S., 1994. "Measurement and prediction of flow in annular s-shaped ducts". Experimental thermal and fluid science, 9(2), pp. 197-205.

[7] Bailey, D., Britchford, K., Carrote, J., and Stevens, S., 1997. "Performance assessment of an annular s-shaped duct". Journal of Turbomachinery, 119(1), pp. 149-156.

[8] Walker, A., Barker, A., and Carrotte, J., 2011. "Numerical design and experimental evaluation of an aggressive s-shaped compressor transition duct with bleed". In GT2011-45628 ASME 2011 Turbo Expo: Turbine Technical Conference and Exposition, American Society of Mechanical Engineers Digital Collection, pp. 151-161.

[9] Sonoda, T., Arima, T., and Oana, M., 1999. "The Effect of Inlet
Boundary Layer Thickness on the Flow Within an Annular S-Shaped Duct". Journal of Turbomachinery, 121(3), 07, pp. 626-634.

[10] Karakasis, M. K., Naylor, E. M., Miller, R. J., and Hodson, H. P., 2010. "The effect of an upstream compressor on a non-axisymmetric s-duct". In GT2010-23404 ASME Turbo Expo 2010: Power for Land, Sea, and Air, American Society of Mechanical Engineers Digital Collection, pp. 477-486.

[11] Stürzebecher, T., Goinis, G., Voss, C., Sahota, H., Groth, P., and Hammer, S., 2018. "Automated aerodynamic optimization of an aggressive s-shaped intermediate compressor duct". In GT2018-75184 ASME Turbo Expo 2018: Turbomachinery Technical Conference and Exposition, American Society of Mechanical Engineers Digital Collection.

[12] Walker, A. D., Barker, A., Mariah, I., Peacock, G., Carrotte, J. F., and Northall, R., 2014. "An aggressive s-shaped compressor transition duct with swirling flow and aerodynamic lifting struts". In GT2014-25844 ASME Turbo Expo 2014: Turbine Technical Conference and Exposition, American Society of Mechanical Engineers Digital Collection.

[13] Bergstedt, R., Walker, D., Peacock, G., and Wallin, F., 2015. "Aerodesign and validation of turning struts for an intermediate compressor duct". In The 22nd International Symposium on Air Breathing Engines (ISABE), Phoenix, AZ, Oct, American Institute of Aeronautics and Astronautics, pp. 25-30.

[14] Clark, C. J., Pullan, G., Curtis, E., and Goenaga, F., 2017. "Secondary flow control in low aspect ratio vanes using splitters". Journal of Turbomachinery, 139(9), p. 091003.

[15] Spalart, P., and Allmaras, S., 1992. "A one equation turbulence model for aerodynamic flows.". AIAA Journal, 94-439, pp.-.

[16] Brandvik, T., and Pullan, G., 2010. "An accelerated 3D Navier Stokes solver for flows in turbomachines". ASME J. Turbomach., 133(2), Oct., pp. 021025-021025.

[17] Grimshaw, S., and Taylor, J., 2016. "Fast settling millimetrescale five-hole probes". In GT2016-56628 ASME Turbo Expo 2016: Turbomachinery Technical Conference and Exposition, American Society of Mechanical Engineers Digital Collection.

[18] Glover, F., 1989. "Tabu search part i”. ORSA Journal on computing, 1(3), pp. 190-206.

[19] Denton, J. D., 1993. "The 1993 igti scholar lecture: Loss mechanisms in turbomachines". ASME J. Turbomach., 115(4), Oct., pp. 621-656.

[20] Hercock, R. \& Williams, D. D., 1974. "Aerodynamic response, distortion induced engine instability". AGARD Lecture Series. 